\title{
Cardiovascular drug utilization post-implant is related to clinical outcome in heart failure patients receiving cardiac resynchronization therapy
}

\author{
Zoltan Bakos ${ }^{1}$, Christian Reitan ${ }^{1}$, Anna Werther-Evaldsson ${ }^{2}$, \\ Anders Roijer ${ }^{2}$, Pyotr Platonov ${ }^{1}$, Rasmus Borgquist ${ }^{1}$ \\ ${ }^{1}$ Arrhythmia Clinic, Skane University Hospital, Lund University, Lund, Sweden \\ ${ }^{2}$ Clinic for Heart Failure and Valvular Disease, Skane University Hospital, Lund University, Lund, Sweden
}

\begin{abstract}
Background: In select patients with heart failure, cardiac resynchronization therapy (CRT) is the most common complementary treatment besides medical treatment. We aimed to assess the association between post CRT-implant changes in cardiovascular medication and cardiovascular mortality and heart failure hospitalization.

Methods: 211 patients on optimal medical therapy eligible for CRT were retrospectively included in this study (72 \pm 7 years, $80 \%$ male, 66\% left bundle branch block, 48\% dilated cardiomyopathy and investigated at baseline and after 6 months. Follow-up with medication, biochemical markers and echocardiography was performed and 3-year mortality data was collected.

Results: At 6 months post-implant the cohort was divided into two groups; 157 patients had low dosage furosemide treatment (up to $40 \mathrm{mg}$ ) and 54 patients were treated with high dosage ( $>40 \mathrm{mg}$ ). A composite endpoint of heart failure hospitalization and all-cause mortality was evaluated at 30 months $(881 \pm 267$ days) after the 6 -month visit. In multivariate Cox regression analysis, $p a-$ tients in the high dose diuretics group had a higher risk of the primary endpoint (HR 1.9 [1.1-3.4], $p=0.033)$, but treatment with high dose diuretics was not associated with improved clinical symptoms $(r=0.031, p=0.64)$.

Conclusions: High dosage of loop-diuretics was associated with worse medium-term clinical outcome in CRT treated patients. It is unclear whether there is a direct causality between these associations, or if higher prescribed dosage of loop-diuretics is just a marker of more severe disease. Higher dose loop diuretics do not necessarily improve the symptoms and may be harmful to the patient. Prospective trials are warranted to further elucidate these findings. (Cardiol J 2017; 24, 4: 374-384)
\end{abstract}

Key words: cardiac resynchronization therapy, heart failure, dilated cardiomyopathy, ischaemic heart failure, medical therapy, loop diuretics

\section{Introduction}

Heart failure (HF) is a common cause of death in developed countries [1]. In the last decade cardiac resynchronization therapy (CRT) has emerged as a complement to medical therapy in patients with wide QRS and impaired left ventricular ejection fraction
$[2,3]$. Large randomized trials have demonstrated the advantage of CRT, however $30-40 \%$ of the patients have no perceived benefit; so called non-responders. The reason for non-response seems to be multifactorial, and data from different trials suggest that patient selection, electrode placement, and optimization of therapy at follow-up all play a role [4-10]. Medical

Address for correspondence: Zoltan Bakos, MD, Arrhythmia Clinic, Skane University Hospital, Getingevagen, 22185 Lund, Sweden, tel: +4646174281, e-mail: zbakos@hotmail.com 
therapy with beta-blockers, angiotensin converting enzyme inhibitors (ACEI) or angiotensin-receptor blockers (ARBs) and aldosterone antagonists [11-13] are all important in HF treatment with proven long term benefits in terms of morbidity and mortality [14]. Aldosterone receptor inhibitors are also an important part of the medical therapy; however, they are less-tolerated mainly due to the elevated serum potassium level [15]. Angiotensin receptor blocker with neprilysin inhibitor (ARNI), as a new medical treatment is on the horizon for HF population, however the long-term effect of the treatment is unknown, and there are no data about this therapy in the CRT population [16]. The potential risks and benefits by changing the medication for CRT-treated patients are less documented, and it is still unclear to what extent changes in medical therapy can influence clinical outcome in this subpopulation. Data regarding medication changes and prognostic outcome is lacking for CRT-treated patients outside the clinical trial setting, but could add knowledge concerning clinical prognosis for these patients. A few studies have suggested that loop diuretic therapy is associated with higher mortality in non-CRT HF patients [17], but only one prospective study has evaluated this in a HF population with mild symptoms after CRT implantation [18]. The aim in this study was to assess the association between the efficacy and clinical outcome of CRT in relation to concurrent use of $\mathrm{HF}$ medications, more specifically loop-diuretic therapy due to its ambiguous effect on morbidity and mortality.

\section{Methods}

Consecutive patients receiving CRT in a tertiary Swedish centre between 2011 and 2014 were included retrospectively. All patients had indications for CRT according to the European Society of Cardiology (ESC) recommendations [19, 20]. Patients were on HF therapy with the highest tolerable dosage of beta-blocker and ACEI/ARB prior to implantation. Mortality data up to 4 years was extracted from the Swedish National Cause of Death Registry. Clinical data was evaluated at 6-month post-implant, and retrospectively collected for baseline parameters. Prior to implantation, all patients had an assessment of their preoperative functional clinical status. Subjective clinical improvement and New York Heart Association (NYHA) classification was assessed by the study physician 6 months after the implantation. A subset of patients had available preoperative and follow up data from standardized HF questionnaires (Minne- sota living with heart failure questionnaires [ML$\mathrm{HFQ]}$ ) and quality of life questionnaires (EQ5D). Medical information, 12-lead electrocardiogram (ECG), laboratory examinations and a standardized echocardiography protocol was extracted from the local electronic medical record system. Written informed consent was collected before the enrolment and the local ethics committee approved the study.

\section{Medication}

Information about beta-blockers, ACEI, ARBs, statins, diuretics, aldosterone inhibitors, thrombocyte aggregation inhibitors, oral anticoagulation drugs were collected at baseline and 6 months. Changes in dosages and between substances were recorded, which was initiated under the supervision of the treating physician. Optimal medical therapy was considered as the highest tolerable dosage of beta-blocker and ACEI or ARB as criteria for CRT treatment. Loop diuretic therapy was indicated according to the ESC recommendation [14] and the decision making about changes in medication over time was at the discretion of the treating physicians.

\section{Heart failure symptom evaluation}

Minnesota living with heart failure questionnaires (MLHFQ) [21-23] and standardized instrument for use as a measure of health outcome (EQ5D) [24] formularies and Self Rated Health (EQ VAS) questionnaires were filled in and collected at baseline and 6 months after the implantation. NYHA classification at baseline and at the 6 month visit was evaluated and the enrolled study subjects were directly asked about subjective improvement.

\section{Echocardiography}

Preoperative and at 6-month echocardiography was performed in all patients. Off-line analysis was performed with Echopac BT12 software (Echopac BT12, GE Medical, Hortens, Norway). Left ventricular (LV) volumes and ejection fraction (EF) were measured with Simpson's biplane method [25]. Left ventricular end-diastolic volume (LVEDV) and left ventricular end-systolic volume (LVESV) were measured, and LVESV index were calculated (LVESVi) [26-30]. Mitral regurgitation (MR) was graded 0-3 according to current guidelines [31]. Septal to posterior wall motion delay was measured as the shortest interval between the maximal displacement of the septum and the posterior wall in milliseconds using 2-dimensional papillary muscle level short axis view [32]. 


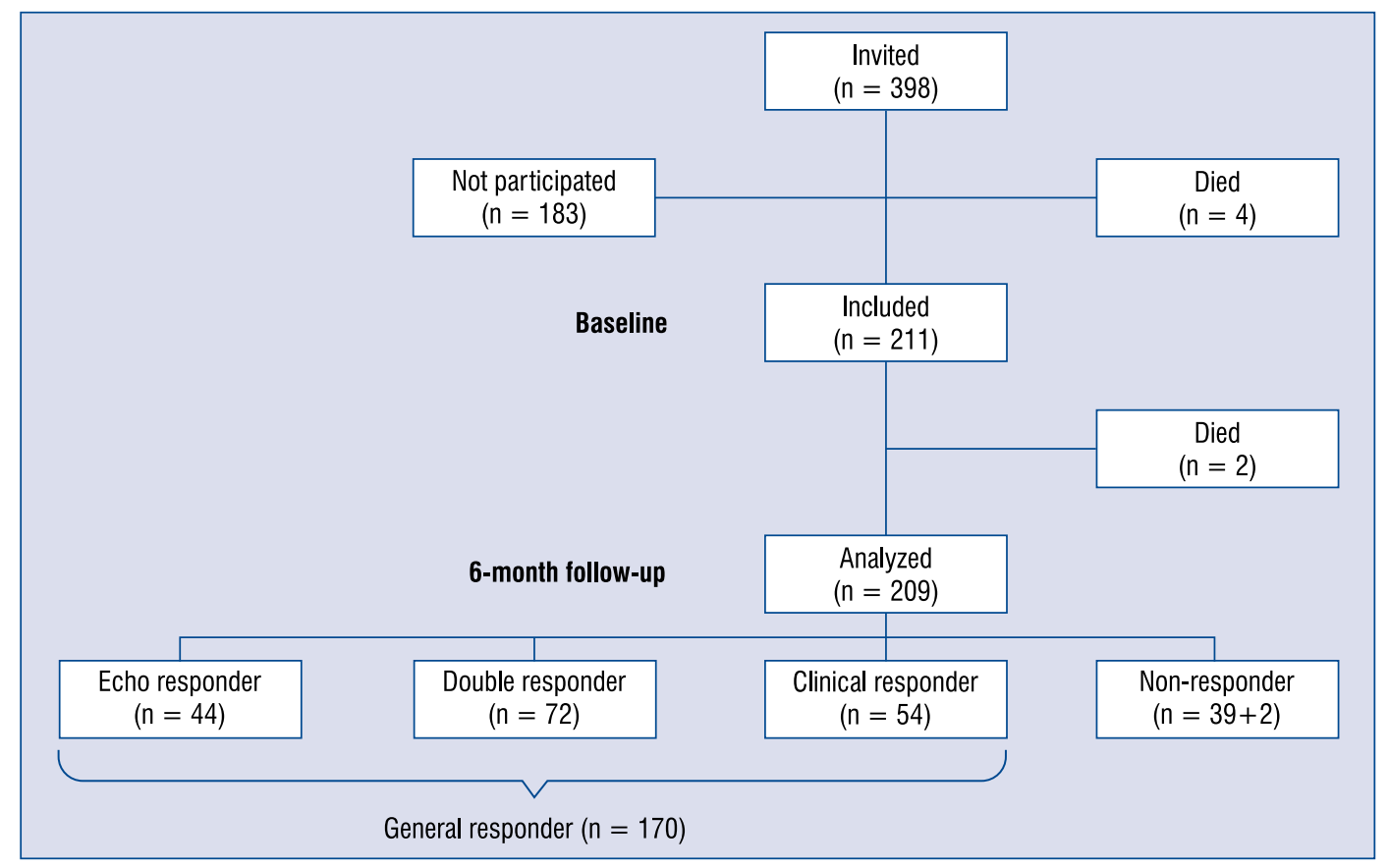

Figure 1. The flow chart demonstrates the inclusion process and the response to the cardiac resynchronization therapy. The different type of response are also represented on the figure.

\section{Definition of positive response to CRT}

At least $15 \%$ reduction or more of the LVESV [33] was considered as reverse remodeling and positive echocardiographic response to the therapy. Positive clinical response was defined as $\geq 1$ NYHA class improvement.

\section{Device implantation and follow up}

Device implantation was performed using left subclavian vascular access. A lateral or posterolateral coronary sinus branch was targeted for the LV lead positioning. The majority of patients $(n=128)$ received a St. Jude Quickopt ${ }^{\oplus}$-algorithm capable device using the algorithm recommended settings. The rest of the patients $(n=83)$ received a Medtronic device, with fixed atrio-ventricular delays of $120 / 150 \mathrm{~ms}$ and simultaneous VV-times.

\section{End point of the study}

The primary endpoint of the present study was death, and secondary endpoint was death or hospitalization for HF. The diagnosis of HF was based either on the medical records or on the record linkage with the national Swedish Patient Registry, if the primary cause of hospitalization was listed as HF.

\section{Statistical analyses \\ SPSS statistical software was used for data analy- sis (IBM, SPSS ver.: 21. 2012). Continuous variables}

are expressed as means ( \pm standard deviation [SD]), categorical variables are presented as frequencies and percentages. For non-normally distributed continuous variables the median value with interquartile range (IQR) is presented. Differences between groups were assessed using paired and unpaired Student t-tests for continuous variables, Mann-Whitney $\mathrm{U}$ test for variables with non-Gaussian distribution, and the $\chi^{2}$ test for categorical variables, or the Fisher's exact test for unordered categorical variables as appropriate. For survival analysis Kaplan-Meier method with log-rank test were used to analyze the cumulative events. Cox regression analysis was used to determine the Hazard ratio (HR) with $95 \%$ confidence interval (CI) for clinical endpoints. The proportional hazards assumption was tested by $\log$ minus log curves and was met. Variables with a $\mathrm{p}<0.10$ were then entered into a multivariate model. Pearson's $r$ test was performed to calculate the correlation coefficient. A two-sided p-value $<0.05$ was considered statistically significant.

\section{Results}

\section{Clinical characteristics of the overall study population}

The group of 398 patients were invited and 211 of these were enrolled. The inclusion process is summarized in Figure 1. The included patients were divided in two subgroups, depending the dosage of 
Table 1. Study population characteristics. The first column represents the whole study population. The second and third columns show the two groups with low and high dose diuretics. The last column shows the p-value for difference between the subgroups.

\begin{tabular}{lcccc}
\hline & Overall & Group I $(\mathbf{n}=\mathbf{1 5 7})$ & Group II $\mathbf{n}=\mathbf{5 4})$ & $\mathbf{P}$ \\
\hline Age [years] \pm SD & $71.6 \pm 7$ & $71.5 \pm 8$ & $71.6 \pm 8$ & 0.96 \\
QRS duration [ms] & 164 & 163 & 166 & 0.29 \\
Male & $80 \%$ & $80 \%$ & $85 \%$ & 0.37 \\
Ischemic cardiomyopathy & $52 \%$ & $47 \%$ & $68 \%$ & $\mathbf{0 . 0 1}$ \\
Hypertension & $59 \%$ & $55 \%$ & $74 \%$ & $\mathbf{0 . 0 2}$ \\
Presence of diabetes & $23 \%$ & $19 \%$ & $35 \%$ & $\mathbf{0 . 0 2}$ \\
Renal failure & $13 \%$ & $11 \%$ & $18 \%$ & 0.16 \\
History of AF & $50 \%$ & $48 \%$ & $61 \%$ & 0.12 \\
History of CABG & $29 \%$ & $26 \%$ & $41 \%$ & 0.06 \\
History of MI & $52 \%$ & $46 \%$ & $68 \%$ & $\mathbf{0 . 0 1}$ \\
CRT-P & $24 \%$ & $24 \%$ & $24 \%$ & 1 \\
LBBB/Non LBBB/PM & $66 \% / 18 \% / 16 \%$ & $67 \% / 19 \% / 14 \%$ & $57 \% / 20 \% / 22 \%$ & 0.31 \\
Echo responder & $62 \%$ & $59 \%$ & $65 \%$ & 0.47 \\
Clinical responder & $58 \%$ & $61 \%$ & $54 \%$ & 0.42 \\
\hline
\end{tabular}

AF — atrial fibrillation; CABG — coronary artery bypass surgery; CRT-P — cardiac resynchronization therapy without defibrillator function; ICMP — ischemic cardiomyopathy; LBBB — left bundle branch block; MI — myocardial injury/infarction; PM — pacemaker rhythm; $\mathrm{SD}$ - standard deviation

Table 2. Medical therapy for the two groups at baseline and at 6 months (\%). The last column shows the $p$-value for difference between the subgroups.

\begin{tabular}{lccc}
\hline Medication (in \%) & Group I ( $\mathbf{n}$ 157) & Group II ( $\mathbf{n}=\mathbf{5 4})$ & P \\
\hline Baseline & & & \\
Beta-blocker & 87 & 92 & 0.33 \\
ACEI/ARB & 96 & 93 & 0.28 \\
Digoxin & 12 & 13 & 0.76 \\
Statin therapy & 60 & 70 & 0.56 \\
Aldosterone antagonist & 49 & 65 & 0.09 \\
Thiazide diuretics & 4 & 5 & 0.65 \\
6-month follow up & & & \\
Beta-blocker & 86 & 92 & 0.41 \\
ACEl/ARB & 89 & 89 & 0.29 \\
Digoxin & 11 & 12 & 0.26 \\
Statin therapy & 56 & 72 & 0.40 \\
Aldosterone antagonist & 45 & 76 & $\mathbf{0 . 0 1}$ \\
Thiazide diuretics & 4 & 5 & 0.62 \\
\hline
\end{tabular}

ACEI — angiotensin converting enzyme inhibitor; ARB - angiotensin receptor blocker

the loop diuretics at 6-month follow up. Based on previous studies, the cut-off value between low and high dosage of furosemide was set at $40 \mathrm{mg}$ [17]. In the low dose group (Group I) the patients had loop diuretic treatment with furosemide up to $40 \mathrm{mg}$ $(\mathrm{n}=157)$. Patients with $>40 \mathrm{mg}$ furosemide were classified as high dose group (Group II, $\mathrm{n}=54$ ). The baseline characteristics of the whole cohort and the two subgroups are presented in Table 1.

The medical therapy initially included beta-blocker in $89 \%$, ACEI/ARB in $96 \%$ and aldosterone blockade in $58 \%$ of the patients. At 6 months, $87 \%$ were on a beta-blocker, $92 \%$ on an ACEI/ARB and $53 \%$ were on aldosterone therapy. Table 2 shows 
information about the HF therapy, comparing baseline and 6-month visit data: At baseline, 68\% of the patients were on loop diuretic therapy, and at 6 months the corresponding percentage was $62 \%$ on loop diuretic therapy $(\mathrm{p}=0.006)$. In Group I the percentage of patients with loop diuretic therapy decreased significantly ( $58 \%$ preoperatively vs. $49 \%$ at 6 -month follow-up, $\mathrm{p}=0.002$ ), however similar changes were not observed in Group II (96\% at baseline vs. $100 \%$ at 6 -month follow-up, $\mathrm{p}=0.32$ ). The mean furosemide dose in Group I pre-implant was $42 \pm 17 \mathrm{mg}$, which was decreased to $36 \pm 7 \mathrm{mg}(\mathrm{p}=0.01)$ at 6 months. In Group II the initial mean dosage of the furosemide was $100 \pm 65 \mathrm{mg}$ at baseline, and it increased to $115 \pm$ $\pm 85 \mathrm{mg}$ at 6 months $(\mathrm{p}=0.11)$.

\section{Biochemical markers}

At baseline, slightly elevated creatinine in Group II was observed compared with Group I $(124 \pm 49 \mu \mathrm{mol} / \mathrm{L}$ vs. $103 \pm 60 \mu \mathrm{mol} / \mathrm{L}, \mathrm{p}=0.09)$ and at 6 months, the difference became significant $(126 \pm 58 \mu \mathrm{mol} / \mathrm{L}$ vs. $108 \pm 50 \mu \mathrm{mol} / \mathrm{L}, \mathrm{p}=0.03)$. However, the change of the creatinine level from baseline to 6 -months within the respective groups did not differ significantly $(\mathrm{p}=0.16, \mathrm{p}=0.44)$. The N-terminal pro B-type natriuretic peptide (NT-proBNP) in Group II improved significantly (from median $3848 \mathrm{ng} / \mathrm{L}$ [IQR: 3575] to $2039 \mathrm{ng} / \mathrm{L}$ [IQR: 2311], $\mathrm{p}=0.03$ ) as well as in Group I (from $3695 \mathrm{ng} / \mathrm{L}$ [3581] to $2740 \mathrm{ng} / \mathrm{L}$ [2427], $\mathrm{p}=0.05$ ). However, no significant difference at baseline $(\mathrm{p}=0.73)$ or at follow-up $(\mathrm{p}=0.69)$ was found in NT-proBNP levels, between the two groups.

\section{Association between cardiovascular medication and clinical improvement}

Self-assessed quality of life pre-implant (1-100 scale) was similar in the two groups $(57 \pm 21$ vs. $59 \pm 20, \mathrm{p}=0.78$ ). However, at the 6 -month follow-up, patients in Group I had a better overall subjective health assessment compared to patients in Group II ( $69 \pm 18$ vs. $62 \pm 20, p=0.03)$. The mean NYHA classification at baseline was similar in both subgroups. Sixty-six percent of the patients showed improvement in NYHA class and the improvement in both groups were similar during the 6 -month follow-up, as presented in Table 3 $(\mathrm{p}<0.01$ in both group). Improvement of the NYHA class was neither correlated to aldosterone antagonist therapy $(\mathrm{r}=0.08, \mathrm{p}=0.25)$, nor to loop diuretic treatment $(\mathrm{r}=0.03, \mathrm{p}=0.64)$ or betablocker therapy ( $\mathrm{r}=0.001, \mathrm{p}=0.99)$ at 6 -months. No correlation was observed between baseline beta-blocker $(\mathrm{r}=0.03, \mathrm{p}=0.62)$, aldosterone inhibitor $(\mathrm{r}=0.03, \mathrm{p}=0.61)$, or loop diuretic $(r=0.04, p=0.54)$ therapy and clinical improvement. In contrast, clinical improvement did show a correlation with baseline ACEI/ARB therapy $(\mathrm{r}=0.17, \mathrm{p}=0.01)$ and 6 month ACEI/ARB therapy $(r=0.15, p=0.03)$.

\section{Association between cardiovascular medication and reverse remodeling}

Sixty-one percent of the patients $(n=128)$ showed more than $15 \%$ reduction in the LVESV (64\% in Group I, $56 \%$ in Group II, $p=0.37$ ). The overall mean LVEF improved in the whole population (from $27 \pm 8 \%$ to $35 \pm 13 \% \mathrm{p}=0.001$ ). Correlations between reverse remodeling and $\mathrm{HF}$ medications were tested. Reduction in LVESV showed a positive trend in correlation with baseline beta-blocker $(r=0.117, p=0.049)$ and ACEI $(\mathrm{r}=0.283, \mathrm{p}=0.066)$ therapy. However, diuretic therapy with furosemide $(\mathrm{r}=0.042, \mathrm{p}=0.58)$ or aldosterone inhibitors $(r=0.047, p=0.54)$ was not associated with reverse remodeling. Improvement of the LVEF showed no correlation with the loop diuretic therapy $(r=1, p=0.38)$ or with aldosterone antagonist treatment $(\mathrm{r}=0.08, \mathrm{p}=0.3)$. The mitral regurgitation improved during this 6 month follow up ( $\mathrm{p}<0.01$ ). Initially 28 patients had grade II and only 2 patients had grade III mitral regurgitation. At 6 month control the mitral regurgitation had improved, only 6 patients had grade II regurgitation, and only 1 patient had grade III regurgitation due to mitral prolapse. The improvement of the regurgitation was not correlated with the dose of the diuretic therapy $(r=0.02, p=0.85)$.

\section{Association between cardiovascular medication and clinical endpoints}

During the whole follow up period $(826 \pm 331$ days) 26 deaths and 27 hospitalizations for $\mathrm{HF}$ were observed. 14 patients were hospitalized due to $\mathrm{HF}$ and 2 patients died before the 6 -month follow up visit in sudden cardiac death despite CRT-D, and these events were excluded from the outcome analyses. During the remainder of the followup, 13 deaths occurred in Group I and 11 deaths were observed in Group II. The effect on clinical outcome of medical therapy using ACEI/ARBs, beta-blockers or aldosterone antagonists was examined by univariate Cox regression analysis. In the univariate Cox regression analysis, high dose loop diuretic therapy was associated with a HR of 1.94 (95\% CI 1.11-3.376). Accordingly, in Kaplan-Meier analysis a significant difference was found 
Table 3. Biochemical marker levels, clinical symptoms and echocardiographic parameters for the two groups at baseline and at 6 months. The last column shows the $p$-value for difference between the subgroups. The $\Delta$-values show the mean ( \pm standard deviation) difference between the baseline and 6-month values of each parameter.

\begin{tabular}{|c|c|c|c|}
\hline Variable & Group I $(n=157)$ & Group II $(n=54)$ & $\mathbf{P}$ \\
\hline \multicolumn{4}{|l|}{ Biochemistry } \\
\hline Baseline creatinine $[\mu \mathrm{mol} / \mathrm{L}]$ & $103 \pm 89$ & $124 \pm 49$ & 0.09 \\
\hline 6-month creatinine $[\mu \mathrm{mol} / \mathrm{L}]$ & $108 \pm 50$ & $126 \pm 58$ & 0.03 \\
\hline Baseline hemoglobin [g/L] & $136 \pm 14$ & $132 \pm 16$ & 0.12 \\
\hline 6-month hemoglobin [g/L] & $137 \pm 13$ & $135 \pm 16$ & 0.34 \\
\hline Baseline NT-proBNP [ng/L] & $3615 \pm 5617$ & $3950 \pm 5384$ & 0.73 \\
\hline 6-month NT-proBNP [ng/L] & $2646 \pm 5295$ & $2314 \pm 2950$ & 0.69 \\
\hline \multicolumn{4}{|l|}{ Clinical markers } \\
\hline Baseline MLWHF & $40 \pm 20$ & $44 \pm 25$ & 0.55 \\
\hline 6-month MLWHF & $27 \pm 20$ & $39 \pm 24$ & 0.001 \\
\hline Baseline EQ5D & $5.7 \pm 3.7$ & $5.4 \pm 3.8$ & 0.76 \\
\hline 6-month EQ5D & $6.1 \pm 2.5$ & $6.2 \pm 3$ & 0.75 \\
\hline Baseline EQ-VAS & $57 \pm 21$ & $59 \pm 20$ & 0.78 \\
\hline 6-month EQ-VAS & $69 \pm 18$ & $62 \pm 20$ & 0.03 \\
\hline NYHA baseline & $2.4 \pm 0.8$ & $2.4 \pm 0.8$ & 0.95 \\
\hline 6-month NYHA & $1.7 \pm 0.6$ & $1.7 \pm 0.7$ & 0.76 \\
\hline \multicolumn{4}{|l|}{ Echocardiography } \\
\hline Baseline LVESV BP [mL] & $141 \pm 53$ & $173 \pm 81$ & 0.03 \\
\hline Baseline LVEDV BP [mL] & $196 \pm 65$ & $228 \pm 92$ & 0.02 \\
\hline Baseline LVEF BP [mL] & $27 \pm 8$ & $25 \pm 8$ & 0.14 \\
\hline 6-month LVESV BP [mL] & $112 \pm 52$ & $141 \pm 85$ & 0.03 \\
\hline 6-month LVEDV BP [mL] & $164 \pm 60$ & $205 \pm 97$ & 0.01 \\
\hline 6-month LVEF BP [mL] & $36 \pm 15$ & $33 \pm 8$ & 0.23 \\
\hline Baseline mitral regurgitation & $1 \pm 0.57$ & $1.1 \pm 0.51$ & 0.80 \\
\hline 6-month mitral regurgitation & $0.81 \pm 0.51$ & $0.86 \pm 0.46$ & 0.62 \\
\hline \multicolumn{4}{|c|}{ Difference between the baseline and 6-month values } \\
\hline$\Delta$ LVESV BP [mL] & $32 \pm 40$ & $32 \pm 50$ & 0.97 \\
\hline$\Delta$ LVEDV BP $[\mathrm{mL}]$ & $32 \pm 51$ & $25 \pm 58$ & 0.40 \\
\hline$\Delta$ LVEF BP [mL] & $8 \pm 15$ & $7 \pm 9$ & 0.48 \\
\hline
\end{tabular}

BP — biplane view; LVEF — left ventricular ejection fraction; LVEDV — left ventricular end diastolic volume; LVESV - left ventricular end systolic volume; MLFHQ - Minnesota living with heart failure questionaires; NT-proBNP — N-terminal pro B-type natriuretic peptide

between the two groups ( $\mathrm{p}=0.03$; Fig. 2). Absence of beta-blocker or aldosterone antagonist therapy showed no influence on the primary endpoint using univariate analysis, but ACEI/ARB therapy significantly reduced risk $(\mathrm{HR}=0.35,95 \% \mathrm{CI}$ $0.123-0.988, p=0.04)$. In the multivariate Cox regression model, high dose diuretic therapy adjusted for age, sex, aetiology, renal disease, showed a HR of $2.1(95 \%$ CI $1.0-4.2, \mathrm{p}=0.04)$ for the primary endpoint. None of the other variables, such as ischemic etiology of $\mathrm{HF}$ ( $\mathrm{p}=0.82, \mathrm{HR}=0.93,95 \%$ CI 0.49-1.78), renal disease ( $\mathrm{p}=0.57, \mathrm{HR}=1.27$,
95\% CI 0.553-2.91), or echocardiographic response ( $\mathrm{p}=0.96, \mathrm{HR}=0.98,95 \%$ CI $0.57-1.7)$ showed independent predictive value for clinical outcome.

Association between diuretic therapy and cardiac device type was noted. Fifty patients received CRT without defibrillator function (CRT-P) device and the majority of the population $(n=161)$ had CRT with defibrillator function (CRT-D) implanted. The distribution of CRT-D therapy was similar in Group I and Group II (75\% vs. $74 \%$, $\mathrm{p}=0.9$ ). As expected, the CRT-P patients were older (76 \pm 9 vs. $70 \pm 9$ years, $p=0.001)$, but had sim- 


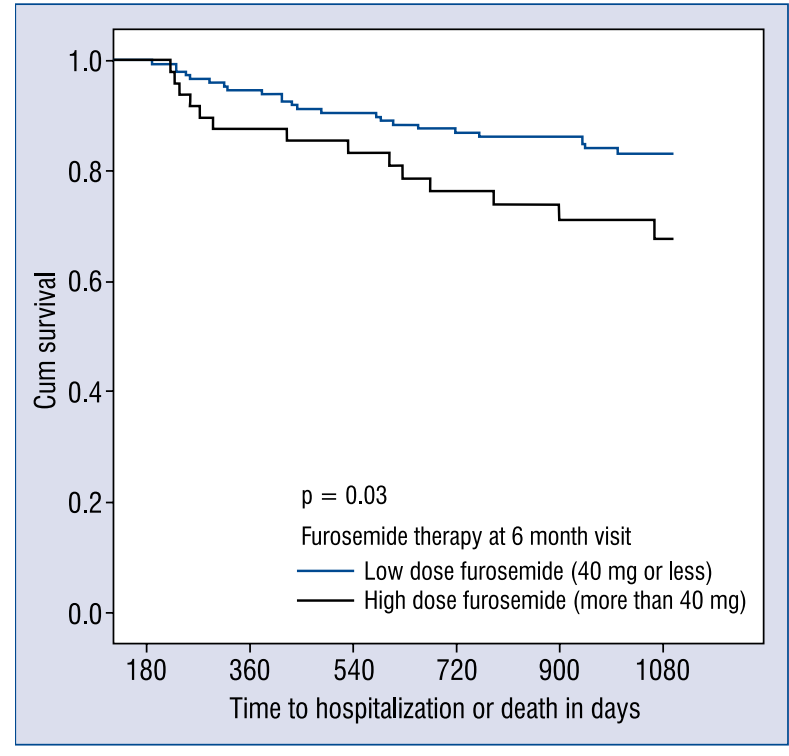

Figure 2. Kaplan-Meier curve is showing the event free survival in patients with low (Group I) and high (Group II) dose diuretics respectively. The curve is a landmark analysis reset at the 6-month follow-up visit.

ilar NYHA class ( $2.6 \pm 0.5$ vs. $2.5 \pm 0.6, \mathrm{p}=0.07)$ at baseline. The NYHA class improvement was slightly better in the CRT-D treated patients at 6 months $(1.8 \pm 0.6$ vs. $1.9 \pm 0.6, p=0.055)$. In patients with CRT-D devices, no prognostic difference was found when stratifying for diuretic therapy dosage (Group I: $\mathrm{n}=120$ vs. Group II: $\mathrm{n}=41$, $\mathrm{p}=0.15$ ) regarding composite clinical end points (Fig. 3A), but in patients with CRT-P devices low dose diuretic therapy (Group I: $\mathrm{n}=37$ ) patients had a better survival compared with higher dosages (Group II: $\mathrm{n}=13$ ), $\mathrm{p}=0.02$ (Fig. 3B).

\section{Discussion}

Our main finding in this observational study suggests that higher dosage loop diuretic therapy at follow up is independently associated with increased risk for medium term death or $\mathrm{HF}$ hospitalization. It is unclear whether the diuretic therapy itself creates an unfavourable circumstance or the lack of positive improvement leads to higher dosage treatment for symptom relief. Patients who required higher diuretic treatment were older and had higher prevalence of hypertension, ischemic heart disease and diabetes mellitus. This finding also supports, that more ill patients often require more diuretic therapy, mainly due to dyspnoea or fluid retention.

The survival and clinical benefit of the CRT is well studied, however the risk of unfavourable events with loop diuretics is less studied in this

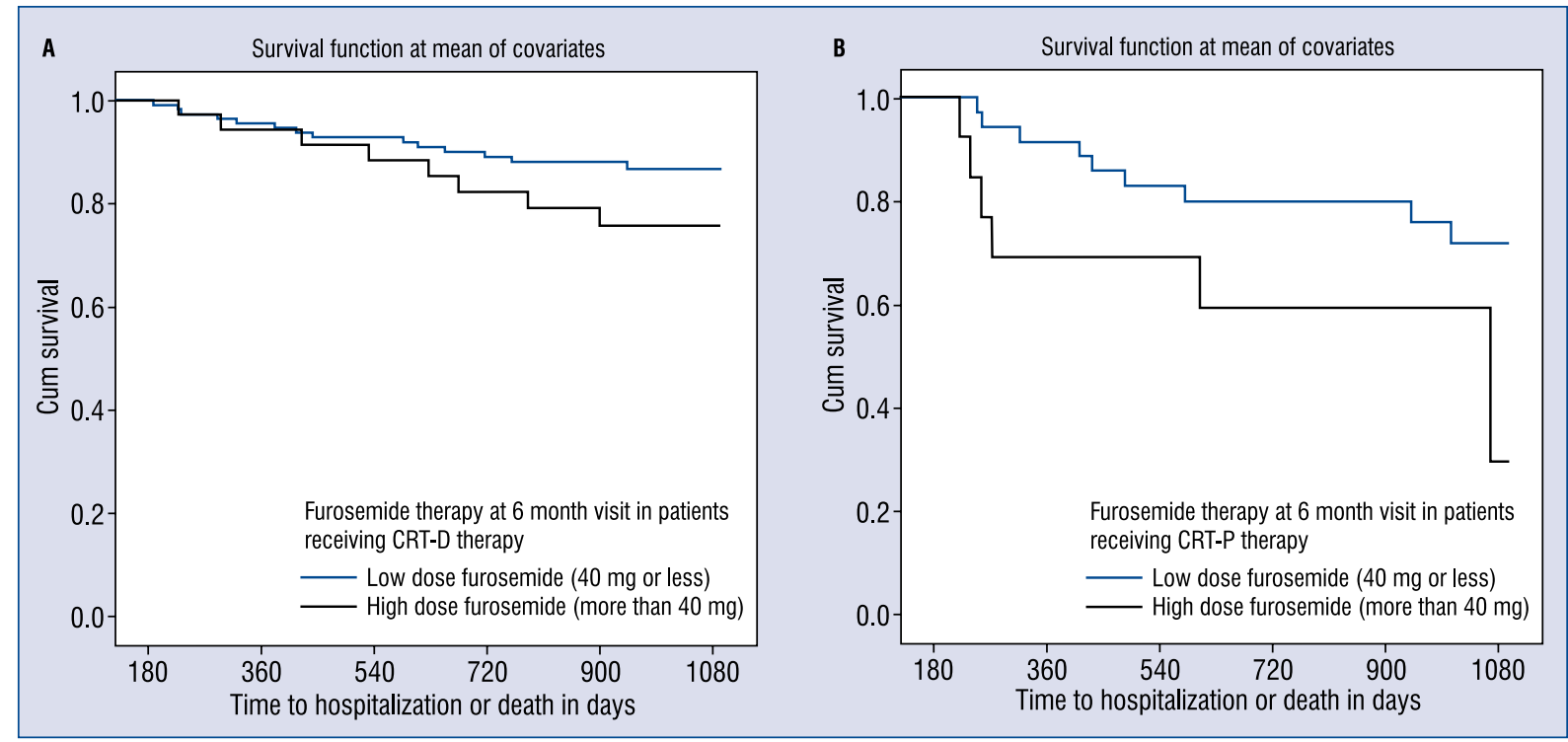

Figure 3. A. Kaplan-Meier demonstrates the event free survival in cardiac resynchronization therapy with defibrillator function (CRT-D) treated patients with low (Group I) and high (Group II) dose diuretics, respectively. The curve is a landmark analysis reset at the 6 month follow-up visit. The difference between the two groups is not significant, $\mathrm{p}=0.15$; B. Kaplan Meier curve demonstrates the event free survival in cardiac resynchronisation therapy without defibrillator function (CRT-P) treated patients with low (Group I) and high (Group II) dose diuretics. The curve is a landmark analysis reset at the six-month follow-up visit. Patients with high loop diuretic therapy has worse outcome, $p=0.02$. 
population. The major finding of this study suggests that the increased diuretic therapy with high dose loop diuretics may be associated with a negative influence on survival and risk of HF hospitalization. The risk for hospitalization/death were more pronounced in the group of CRT-P treated patients, who represented a slightly older population with more comorbidity, compared to the CRT-D treated patients. No positive correlation between loop diuretic dosages and clinical improvement or reverse remodeling was found, regardless of implanted device type.

In "real life", treating the failing heart might differ in some details compared to what is seen in the large clinical trials, even though the long-term benefits of medical and CRT therapy in terms of mortality have been well established by the prospective randomized trials. Depending on the local routines, experiences and possibilities, the optimal medical therapy including dosages may differ.

Routinely, many patients are started on loop diuretics at a time with decompensated HF before the CRT implantation, but over time - and especially post-CRT implant - many of them improve their symptoms including mitral regurgitation [34-37], but nevertheless continue on the same dose of diuretics and are thus treated with a higher dose than necessary. On the other hand, HF therapy is often made complicated by patient specific factors such as low blood pressure, renal dysfunction, and not least by patient preferences and compliance.

Loop diuretic therapy is one of the most commonly used complementary medications for symptom relief and for reducing fluid retention, but long-term data suggests potential harmful effect in higher dosages [17, 38]. Early experimental studies have suggested that in $\mathrm{HF}$ without congestion, furosemide therapy can activate the renin-angiotensin system and thus can influence the HF negatively [39]. An Italian study with 813 ambulatory patients with mild to moderate $\mathrm{HF}$, and found that more than $50 \mathrm{mg}$ furosemide daily increased the 3-year mortality [17]. A recent prospective study with 244 stable non-CRT treated HF patients also found that higher furosemide dose was associated with increased morbidity and mortality, and only $40 \mathrm{mg}$ furosemide daily on top of the standard HF therapy resulted in $66 \%$ increased risk of adverse event [38]. That population was younger and had less comorbidity compared to this present one, but the medical therapy was comparable in both studies. Similar to findings herein, the high dose diuretic therapy also had slightly more comorbidity, higher frequency of renal impairment and more often had ischemic cardiomyopathy and also presented worse outcomes when compared with low dose treatment. Furthermore, Penn et al. [18] used the MADIT-CRT data for investigation and also found that in mild HF patients after CRT implantation, higher diuretic usage was correlated with increased risk of death and hospitalization [18]. However up to date there is no data to support a specific cut-off level for the potentially harmful effects of loop diuretic therapy, and the lack of reduction of the loop diuretic therapy itself can perhaps be considered a potential risk.

Thus, in harmony with other trials, this data also suggests that high dose furosemide therapy may be non-beneficial in the long term [17]. Independently from clinical improvement, renal function or reverse remodeling, higher dose diuretic therapy with furosemide appears to be unfavourable in terms of long term survival. High dose loop diuretic therapy was associated with larger LVESV and LVEDV, but reverse remodeling was similar in both groups and no significant difference in this regard was found between them. The negative influence of the loop diuretic therapy seems to be more prominent in CRT-P recipients who represent an older population with relatively more comorbidity within the CRT group. Loop diuretics may increase the risk of hypokalemia induced malignant arrhythmias and sudden cardiac death, which in theory may explain the difference in outcome for CRT-P treated patients compared to CRT-D. However, of the 26 deaths only two were classified as sudden cardiac death, and these patients both received a CRT-D device.

Together with previously published results, the present data suggest that a high awareness is warranted regarding cardiovascular medication changes for CRT-treated patients. CRT has the potential to induce substantial changes in the cardiac function [40], which in turn may influence blood pressure, renal function and fluid status of the patient. It is imperative to make a renewed assessment of fluid status and loop diuretic need, after the expected (potential) remodelling effect of CRT has taken place, in order to re-evaluate the indication and required dosages for the treatment. For patients where no diuretic reduction is possible, or where an increased dosage of diuretic is required, special attention is warranted. These patients are likely to be at increased risk of $\mathrm{HF}$ 
related morbidity and mortality, and every effort should therefore be made for individual optimization of the CRT-settings and other cardiovascular medications for these patients.

\section{Limitations of the study}

We acknowledge that there are several limitations to this study. It is a single-centre retrospective study, based on registry data. From the invited 398 patients only 211 participated in this trial therefore selection bias cannot be ruled out. All efforts were made to reach the most optimal biventricular pacing, however full data on this was not available for the current analyses. Data about the medication was collected from the local medical database and from the participants but no verification was available for compliance on individual level. Medication changes during the study were at the discretion of the patient's treating physician and the study group had no influence on these changes. Clinical endpoints were collected from the validated Swedish national death and hospitalization registries, which ensures a high accuracy, even though no individual scrutiny of the electronic medical records was possible regarding mortality cause. The reduction of the NT-proBNP - which is an important HF marker - can also be secondary to the improvement of the fluid balance in some patients, this cannot be ruled out. However, all of the study subjects were on optimal medical therapy before the CRT implantation with stable HF status.

\section{Conclusions}

In this observational study, higher dosages of loop diuretic therapy were associated with worse medium-term clinical outcome in CRT treated patients. However, higher doses of loop diuretic therapy showed no influence on echocardiographic remodeling response, or on clinical improvement. It is unclear whether there is a direct causality between these associations, or if higher prescribed dosage of loop-diuretics is just a marker of more severe disease. Prospective trials are needed to further elucidate these findings, and it may be warranted to try to reduce the dosage of loop diuretics in the post-CRT implant phase. In cases, where the response to the treatment is poor and high dose diuretics are necessary, further interventions are desirable to prevent an unfavourable clinical outcome.

Conflict of interest: None declared

\section{References}

1. Mosterd A, Hoes AW. Clinical epidemiology of heart failure. Heart. 2007; 93(9): 1137-1146, doi: 10.1136/hrt.2003.025270, indexed in Pubmed: 17699180.

2. Ponikowski P, Voors AA, Anker SD, et al. Authors/Task Force Members. 2016 ESC Guidelines for the diagnosis and treatment of acute and chronic heart failure: The Task Force for the diagnosis and treatment of acute and chronic heart failure of the European Society of Cardiology (ESC)Developed with the special contribution of the Heart Failure Association (HFA) of the ESC. Eur Heart J. 2016; 37(27): 2129-2200, doi: 10.1093/eurheartj/ ehw128, indexed in Pubmed: 27206819.

3. Brignole M, Auricchio A, Baron-Esquivias G, et al. ESC Committee for Practice Guidelines (CPG), Document Reviewers. 2013 ESC Guidelines on cardiac pacing and cardiac resynchronization therapy: the Task Force on cardiac pacing and resynchronization therapy of the European Society of Cardiology (ESC). Developed in collaboration with the European Heart Rhythm Association (EHRA). Eur Heart J. 2013; 34(29): 2281-2329, doi: 10.1093/ eurheartj/eht150, indexed in Pubmed: 23801822.

4. Stankovic I, Prinz C, Ciarka A, Daraban AM, Kotrc M, Aarones $\mathrm{M}$, et al. Relationship of visually assessed apical rocking and septal flash to response and long-term survival following cardiac resynchronization therapy (PREDICT-CRT). European heart journal cardiovascular Imaging. 2015.

5. Imamura T, Kinugawa K, Nitta D, et al. Complete left bundle branch block and smaller left atrium are predictors of response to cardiac resynchronization therapy in advanced heart failure. Circ J. 2015; 79(11): 2414-2421, doi: 10.1253/circj.CJ-15-0769, indexed in Pubmed: 26376671.

6. Biton Y, Kutyifa V, Zareba W, et al. Long-term outcome with cardiac resynchronization therapy in mild heart failure patients with left bundle branch block from US and Europe MADIT-CRT. Heart Fail Rev. 2015; 20(5): 535-543, doi: 10.1007/s10741-0159499-2, indexed in Pubmed: 26216033.

7. Hartlage GR, Suever JD, Clement-Guinaudeau S, et al. Prediction of response to cardiac resynchronization therapy using left ventricular pacing lead position and cardiovascular magnetic resonance derived wall motion patterns: a prospective cohort study. J Cardiovasc Magn Reson. 2015; 17: 57, doi: 10.1186/ s12968-015-0158-5, indexed in Pubmed: 26170046.

8. Van't Sant J, Ter Horst IAH, Wijers SC, et al. Measurements of electrical and mechanical dyssynchrony are both essential to improve prediction of CRT response. J Electrocardiol. 2015; 48(4): 601-608, doi: 10.1016/j.jelectrocard.2015.01.015, indexed in Pubmed: 25754584.

9. Kydd AC, Khan FZ, Ring L, et al. Development of a multiparametric score to predict left ventricular remodelling and prognosis after cardiac resynchronization therapy. Eur J Heart Fail. 2014; 16(11): 1206-1213, doi: 10.1002/ejhf.167, indexed in Pubmed: 25302484.

10. Zhang H, Dai $Z$, Xiao $P$, et al. The left ventricular lead electrical delay predicts response to cardiac resynchronisation therapy. Heart Lung Circ. 2014; 23(10): 936-942, doi: 10.1016/j. hlc.2014.04.002, indexed in Pubmed: 24996391.

11. Pitt B, Zannad F, Remme WJ, et al. The effect of spironolactone on morbidity and mortality in patients with severe heart failure. Randomized Aldactone Evaluation Study Investi- 
gators. N Engl J Med. 1999; 341(10): 709-717, doi: 10.1056/ NEJM199909023411001, indexed in Pubmed: 10471456.

12. Dhillon S. Eplerenone: a review of its use in patients with chronic systolic heart failure and mild symptoms. Drugs. 2013; 73(13): 1451-1462, doi: 10.1007/s40265-013-0098-z, indexed in Pubmed: 23881669.

13. Zannad F, McMurray JJV, Krum H, et al. EMPHASIS-HF Study Group. Eplerenone in patients with systolic heart failure and mild symptoms. N Engl J Med. 2011; 364(1): 11-21, doi: 10.1056/ NEJMoa1009492, indexed in Pubmed: 21073363.

14. Taylor J. The 2012 ESC Guidelines on Heart Failure. Eur Heart J. 2012; 33(14): 1703-1704, doi: 10.1093/eurheartj/ehs138, indexed in Pubmed: 22797149.

15. Lainscak M, Pelliccia F, Rosano G, et al. Safety profile of mineralocorticoid receptor antagonists: Spironolactone and eplerenone. Int J Cardiol. 2015; 200: 25-29, doi: 10.1016/j.ijcard.2015.05.127, indexed in Pubmed: 26404748.

16. Okumura N, Jhund PS, Gong J, et al. PARADIGM-HF Investigators and Committees*. Effects of Sacubitril/Valsartan in the PARADIGM-HF Trial (Prospective Comparison of ARNI with ACEI to Determine Impact on Global Mortality and Morbidity in Heart Failure) According to Background Therapy. Circ Heart Fail. 2016; 9(9), doi: 10.1161/CIRCHEARTFAILURE.116.003212, indexed in Pubmed: 27618854.

17. Dini FL, Ghio S, Klersy C, et al. Effects on survival of loop diuretic dosing in ambulatory patients with chronic heart failure using a propensity score analysis. Int J Clin Pract. 2013; 67(7): 656-664, doi: 10.1111/ijcp.12144, indexed in Pubmed: 23758444.

18. Penn J, Goldenberg I, McNitt S, et al. Changes in Drug Utilization and Outcome With Cardiac Resynchronization Therapy: A MADIT-CRT Substudy. J Card Fail. 2015; 21(7): 541-547, doi: 10.1016/j.cardfail.2015.03.006, indexed in Pubmed: 25800548 .

19. Dickstein K, Vardas PE, Auricchio A, et al. ESC Committee for Practice Guidelines, Committee for Practice Guidelines of the European Society of Cardiology, ESC Committee for Practice Guidelines (CPG), ESC Committee for Practice Guidelines (CPG). 2010 Focused Update of ESC Guidelines on device therapy in heart failure: an update of the 2008 ESC Guidelines for the diagnosis and treatment of acute and chronic heart failure and the 2007 ESC guidelines for cardiac and resynchronization therapy. Developed with the special contribution of the Heart Failure Association and the European Heart Rhythm Association. Eur Heart J. 2010; 31(21): 2677-2687, doi: 10.1093/eurheartj/ehq337, indexed in Pubmed: 20801924.

20. Brignole M, Auricchio A, Baron-Esquivias G, et al. 2013 ESC Guidelines on cardiac pacing and cardiac resynchronization therapy: The Task Force on cardiac pacing and resynchronization therapy of the European Society of Cardiology (ESC). Developed in collaboration with the European Heart Rhythm Association (EHRA). Europace. 2013; 15(8): 1070-1118, doi: 10.1093/europace/eut206.

21. Rector TS, Cohn JN. Assessment of patient outcome with the Minnesota Living with Heart Failure questionnaire: reliability and validity during a randomized, double-blind, placebo-controlled trial of pimobendan. Pimobendan Multicenter Research Group. Am Heart J. 1992; 124(4): 1017-1025, indexed in Pubmed: 1529875.

22. Guyatt GH. Measurement of health-related quality of life in heart failure. J Am Coll Cardiol. 1993; 22(4 Suppl A): 185A-191A, indexed in Pubmed: 8376691.
23. Höfer S, Lim L, Guyatt G, et al. The MacNew Heart Disease health-related quality of life instrument: a summary. Health Qual Life Outcomes. 2004; 2: 3, doi: 10.1186/1477-7525-2-3, indexed in Pubmed: 14713315.

24. Rabin R, de Charro F. EQ-5D: a measure of health status from the EuroQol Group. Ann Med. 2001; 33(5): 337-343, indexed in Pubmed: 11491192.

25. Schiller NB, Shah PM, Crawford M, et al. Recommendations for quantitation of the left ventricle by two-dimensional echocardiography. American Society of Echocardiography Committee on Standards, Subcommittee on Quantitation of Two-Dimensional Echocardiograms. J Am Soc Echocardiogr. 1989; 2(5): 358-367, indexed in Pubmed: 2698218.

26. Young JB, Abraham WT, Smith AL, et al. Multicenter InSync ICD Randomized Clinical Evaluation (MIRACLE ICD) Trial Investigators. Combined cardiac resynchronization and implantable cardioversion defibrillation in advanced chronic heart failure: the MIRACLE ICD Trial. JAMA. 2003; 289(20): 2685-2694, doi: 10.1001/jama.289.20.2685, indexed in Pubmed: 12771115.

27. Gold MR, Thébault $\mathrm{C}$, Linde $\mathrm{C}$, et al. Effect of QRS duration and morphology on cardiac resynchronization therapy outcomes in mild heart failure: results from the Resynchronization Reverses Remodeling in Systolic Left Ventricular Dysfunction (REVERSE) study. Circulation. 2012; 126(7): 822-829, doi: 10.1161/CIRCULATIONAHA.112.097709, indexed in Pubmed: 22781424.

28. Linde C, Abraham WT, Gold MR, et al. REVERSE (REsynchronization reVErses Remodeling in Systolic left vEntricular dysfunction) Study Group. Randomized trial of cardiac resynchronization in mildly symptomatic heart failure patients and in asymptomatic patients with left ventricular dysfunction and previous heart failure symptoms. J Am Coll Cardiol. 2008; 52(23): 1834-1843, doi: 10.1016/j.jacc.2008.08.027, indexed in Pubmed: 19038680.

29. Sullivan RM, Murillo J, Gerritse B, et al. Do baseline diastolic echocardiographic parameters predict outcome after resynchronization therapy? Results from the PROSPECT trial. Pacing Clin Electrophysiol. 2013; 36(2): 214-220, doi: 10.1111/pace.12042, indexed in Pubmed: 23121136.

30. Killu AM, Grupper A, Friedman PA, et al. Predictors and outcomes of "super-response" to cardiac resynchronization therapy. J Card Fail. 2014; 20(6): 379-386, doi: 10.1016/j.cardfail.2014.03.001, indexed in Pubmed: 24632340.

31. American College of $\mathrm{C}$, American Heart Association Task Force on Practice G, Society of Cardiovascular A, Bonow RO, Carabello BA, Chatterjee K, et al. ACC/AHA 2006 guidelines for the management of patients with valvular heart disease: a report of the American College of Cardiology/American Heart Association Task Force on Practice Guidelines (writing Committee to Revise the 1998 guidelines for the management of patients with valvular heart disease) developed in collaboration with the Society of Cardiovascular Anesthesiologists endorsed by the Society for Cardiovascular Angiography and Interventions and the Society of Thoracic Surgeons. Journal of the American College of Cardiology. 2006;48(3):e1-148.

32. Pitzalis MV, Iacoviello M, Romito R, et al. Cardiac resynchronization therapy tailored by echocardiographic evaluation of ventricular asynchrony. J Am Coll Cardiol. 2002; 40(9): 1615-1622, indexed in Pubmed: 12427414.

33. Doltra A, Bijnens B, Tolosana JM, et al. Mechanical abnormalities detected with conventional echocardiography are associated with response and midterm survival in CRT. JACC Cardiovasc Imaging. 2014; 7(10): 969-979, doi: 10.1016/j.jcmg.2014.03.022, indexed in Pubmed: 25240452. 
34. van Bommel RJ, Marsan NA, Delgado V, et al. Cardiac resynchronization therapy as a therapeutic option in patients with moderate-severe functional mitral regurgitation and high operative risk. Circulation. 2011; 124(8): 912-919, doi: 10.1161/CIRCULATIONAHA.110.009803, indexed in Pubmed: 21810666.

35. Lancellotti P, Mélon P, Sakalihasan N, et al. Effect of cardiac resynchronization therapy on functional mitral regurgitation in heart failure. Am J Cardiol. 2004; 94(11): 1462-1465, doi: 10.1016/j. amjcard.2004.07.154, indexed in Pubmed: 15566929.

36. Verhaert D, Popović ZB, De S, et al. Impact of mitral regurgitation on reverse remodeling and outcome in patients undergoing cardiac resynchronization therapy. Circ Cardiovasc Imaging. 2012; 5(1): 21-26, doi: 10.1161/CIRCIMAGING.111.966580, indexed in Pubmed: 22047983.

37. Uretsky BF, Thygesen K, Daubert JC, et al. Predictors of mortality from pump failure and sudden cardiac death in patients with sys- tolic heart failure and left ventricular dyssynchrony: results of the CARE-HF trial. J Card Fail. 2008; 14(8): 670-675, doi: 10.1016/j. cardfail.2008.06.001, indexed in Pubmed: 18926439.

38. Martins J, Lourenço P, Araújo JP, et al. Prognostic implications of diuretic dose in chronic heart failure. J Cardiovasc Pharmacol Ther. 2011; 16(2): 185-191, doi: 10.1177/1074248410388807, indexed in Pubmed: 21335479.

39. McCurley JM, Hanlon SU, Wei Sk, et al. Furosemide and the progression of left ventricular dysfunction in experimental heart failure. J Am Coll Cardiol. 2004; 44(6): 1301-1307, doi: 10.1016/j. jacc.2004.04.059, indexed in Pubmed: 15364336.

40. Padeletti L, Pieragnoli P, Ricciardi G, et al. Acute hemodynamic effect of left ventricular endocardial pacing in cardiac resynchronization therapy: assessment by pressure-volume loops. Circ Arrhythm Electrophysiol. 2012; 5(3): 460-467, doi: 10.1161/ CIRCEP.111.970277, indexed in Pubmed: 22589286. 